\title{
Correction to: Clathrin-Mediated Endocytosis: Methods and Protocols
}

\author{
Laura E. Swan
}

\section{Correction to:}

Laura E. Swan (ed.), Clathrin-Mediated Endocytosis:

Methods and Protocols, Methods in Molecular Biology, vol. 1847, https://doi.org/10.1007/978-1-4939-8719-1

This book was inadvertently published with the incorrect title as Clathrin-Mediated Endoytosis: Methods and Protocols. This has now been corrected throughout the book to Clathrin-Mediated Endocytosis: Methods and Protocols. 